\title{
RENDIMIENTO ACADÉMICO Y TIC. UNA EXPERIENCIA CON WEBS DIDÁCTICAS EN LA UNIVERSIDAD DE MURCIA.
}

\section{ACADEMIC PERFORMANCE AND ICT. A STUDY WITH EDUCATIONAL WEBSITES AT THE UNIVERSITY OF MURCIA, SPAIN.}

\author{
Ana Belén Mirete Ruiz \\ anabelen.mirete@um.es \\ Dr. Francisco Alberto García Sánchez \\ fags@um.es
}

\begin{abstract}
Universidad de Murcia. Facultad de Educación.
Departamento de Métodos de Investigación y Diagnóstico en Educación. Campus Universitario de Espinardo, 30100, Murcia (España)
\end{abstract}

Se analizan las relaciones entre implicación del alumno en la utilización de webs didácticas de asignatura (a través del número de accesos) y rendimiento académico (calificaciones). Todo ello en 189 alumnos de 3 asignaturas de la Universidad de Murcia. Se comprueba como esa implicación de los estudiantes con la utilización de la web didáctica de su asignatura se relaciona positivamente con sus resultados académicos (analizando los datos tanto con técnicas correlaciónales como con técnica de ANOVA). El resultado supone un paso más allá de la constatación previa existente de la buena acogida que los alumnos hacen de estas herramientas didácticas.

Palabras Clave: TIC, web didáctica, enseñanza universitaria, rendimiento académico.

Connections between the student's involvement in the usage of teaching webs (via access count) and academic performance (grades) are analyzed in 189 students of 3 subjects at the University of Murcia. It can be observed that involvement of students with the use of teaching webs is positively related to their academic performance (data was analyzed using both correlational and ANOVE techniques). This result is a step beyond the previous acknowledgement of the favorable reception of those didactical tools by the students. Keywords: ICT, educational website, university education, academic performance. 


\section{Introducción.}

En la implantación de las nuevas titulaciones de Grado y Máster, muchas han sido las experiencias piloto de adaptación al Espacio Europeo de Educación Superior (EEES) que se han sustentado en el uso de las Tecnologías de la Información y la Comunicación (en adelante TIC) (Marín \& Reche, 2011). Es fácil pensar que las metodologías enfocadas a capacitar al alumno hacia su autogestión encuentran refuerzo si se apoyan en las posibilidades que estas tecnologías ofrecen a los procesos educativos. Ahora bien, tampoco debemos sobrevalorar las posibilidades de las TIC y concebirlas como la panacea a los problemas educativos actuales, aunque sea cierto que nos ofrecen un sustrato adecuado para el conjunto general de cambios que han ido gestándose.

Cuando hablamos de tecnologías, parte del profesorado puede creer que su inclusión en las procesos educativos supone una renovación metodológica innovadora drástica, y que simplemente ello propicia un aumento de la motivación y participación de los estudiantes, facilitando la comprensión de los contenidos y el aprendizaje en general (Domingo \& Fuentes, 2010). Pero para que esto pueda ser realmente así, es necesario superar la mera visión instrumental de las TIC, para centrar esfuerzos en el desarrollo de usos pedagógicamente significativos e innovadores, enfocados realmente a transformar las formas de enseñar y aprender. $\mathrm{Y}$ en ese sentido, es importante empezar a valorar la relación existente entre los recursos TIC puestos a disposición del alumnado y su influencia o no en el rendimiento académico.

Las posibilidades didácticas que ofrecen las TIC a la enseñanza y al aprendizaje no van a depender solo de las características de la tecnología que empleemos. Más bien dependerán de las características didácticas específicas con que hayan sido diseñados esas tecnologías, de las demandas a través de ellas se hagan al alumnado, de las posibilidades que ofrezcan a los docentes y de las actividades que tengan que realizar con ellas los alumnos para resolver las tareas de aprendizaje (Area, 2007; Cabero \& López, 2009). Todo ello sin desmerecer las propiedades que las diferentes herramientas tienen en sí mismas y que, indudablemente, permiten ofrecer a los procesos educativos facilidades para la gestión de la información, flexibilización del tiempo y el espacio, posibilidades de interacción entre los usuarios, etc. Pero en cualquier caso, debemos entender que la inclusión de las TIC en las aulas requiere de una rigurosa planificación, que atienda a las características, usuarios y objetivos del proceso educativo en el cual se insertarán y lleve a seleccionar, en función de ello, la tecnología que más se adecúe a los fines pretendidos.

De acuerdo con este argumento, podemos decir que, para la docencia universitaria, precisamos de TIC que incluyan un importante componente didáctico $\mathrm{y}$, a la vez, resulten versátiles, capaces de ajustarse a las necesidades del nuevo modelo educativo centrado en el aprendizaje y a los intereses particulares del profesor y su asignatura. Bajo esta demanda surgen las webs didácticas de asignatura, entendidas como páginas web pensadas, diseñadas y destinadas a facilitar los procesos de enseñanza y aprendizaje de la asignatura en la que se utilizan sirviendo, a la vez, de guía al estudiante a través de ella (Area, 2003; García Sánchez \& Martínez Segura, 2009; García Sánchez, Martínez 
Segura, Mirete \& Martínez Juárez, 2010; Marqués, 2005).

En nuestra práctica docente, la web didáctica se ha convertido en una herramienta de apoyo a las experiencias de formación presenciales, logrando una mayor flexibilidad en los procesos educativos y permitiendo la adquisición y aplicación de nuevas competencias digitales e informacionales en todos los implicados. Esta concepción de la web didáctica nos acerca a los planteamientos metodológicos del blended learning o aprendizaje mezclado o híbrido, el cual supone una fusión de experiencias de formación cara a cara con metodologías de aprendizaje on-line (Garrison \& Vaughan, 2008; Snart, 2010), beneficiándose de las ventajas de ambos modelos.

En la web didáctica articulamos un espacio común en red, en el que docentes y discentes disponen de un medio de comunicación siempre activo, donde el alumnado encuentra en todo momento aquello que le puede ser de utilidad para superar una asignatura. Su finalidad es facilitar que el estudiante realice un ejercicio de metacognición, a la vez que le ofrecen un acceso a la documentación necesaria para la construcción de su propio aprendizaje y una guía para realizarlo. Estas webs didácticas, que van mucho más allá que un mero repositorio de información de una asignatura, responden a demandas como las formuladas por Tejedor, García-Valcárcel y Parada (2009) cuando afirman que no se trata de elaborar materiales tecnológicamente perfectos, sino pedagógicamente significativos, capaces de generar procesos educativos de calidad para el estudiante.

Desde el nacimiento de los procesos de enseñanza on-line hasta la actualidad, son muchas las investigaciones realizadas para conocer la utilidad que las metodologías virtualizadas tienen para el alumnado (Cabero, Llorente \& Puentes, 2010; Chandra \& Ficher, 2009; Sánchez López, García Sánchez, Martínez Segura \& Mirete, 2012; Shahzad \& Khan, 2010; Tesone \& Ricci, 2008). La rápida evolución de las TIC ha ido modificando el objeto de interés de los estudios realizados, desde analizar la valoración por parte de los estudiantes de experiencias de aprendizaje a distancia mediadas por ordenador, a estudiar situaciones de enseñanza basadas en metodologías combinadas, pasando por el uso de recursos virtuales concretos. No obstante, todos ellos coinciden en señalar la valoración positiva que hacen los estudiantes sobre la inclusión de recursos tecnológicos en sus procesos de aprendizaje.

Nuestros trabajos previos nos han permitido contrastar, de forma específica, la buena acogida que tienen las webs didácticas tanto entre los alumnos (García Sánchez \& Martínez Segura, 2009; Sánchez López et al., 2012), como entre los profesores que empiezan a utilizarlas (García Sánchez \& Martínez Segura, 2009). El presente trabajo se centra en saber si estas webs didácticas están influyendo en los resultados de aprendizaje de los estudiantes, entendidos estos en términos de rendimiento académico. Por ello, hemos analizado las relaciones existentes entre el grado de implicación del estudiante con la web didáctica y su rendimiento académico en la asignatura

\section{Metodología.}

\subsection{Participantes.}

Para la elección de los estudiantes participantes se ha utilizado un muestreo intencional o no probabilístico por juicios 
(Hernández Pina \& Maquilón, 2010), en el que los participantes son elegidos teniendo en cuenta un determinado criterio y garantizando acceder a un grupo representativo cuando la población es pequeña. En esta investigación el criterio que ha determinado la selección de los participantes es que hayan cursado recientemente una asignatura en la que se haya utilizado una web didáctica. Así, los grupos diana han sido seleccionados de entre los adscritos a los profesores responsables de asignaturas implicadas en un Proyecto de Innovación1 (García Sánchez et al., 2010) cuya finalidad fue la implementación de webs didácticas y su evaluación.

Para el estudio que aquí presentamos contamos con un total de 189 participantes pertenecientes a las asignaturas Modelo Integral de Actuación en Atención Temprana (20.9\%), Calidad en los Servicios a la discapacidad (24.6\%) y Biopatología Infantojuvenil y Necesidades Educativas Especiales (54.5\%), todas ellas impartidas en distintos cursos del título de Logopedia de la Universidad de Murcia.

\subsection{Materiales e instrumentos.}

La estructura y características de las webs didácticas empleadas han sido detalladas en estudios previos (García Sánchez \& Martínez Segura, 2009; Sánchez López et al., 2012). Estas webs incluyen apartados como el listado de temas y actividades, un cronograma semanal detallado de lo que se va a trabajar en la asignatura, y acceso a recursos materiales mínimos y complementarios para el desarrollo de la asignatura. Junto a ello está disponible también otra información de interés como competencias a desarrollar, metodología, evaluación, etc. Y como aspecto especialmente relevante, toda la información sobre los temas, actividades y materiales a trabajar aparece siempre acompañada de textos breves que orientan al alumno en su uso y estudio, invitan a la reflexión y al ejercicio de una metacognición sobre el aprendizaje que se está realizando.

Desde el inicio de la asignatura, el alumno dispone en la web didáctica de todos los materiales que va a precisar para cursarla, con orientaciones específicas del profesor para su uso y adecuado aprovechamiento dentro de un aprendizaje autónomo. Además, en las clases presenciales el profesor realiza con frecuencia referencias a la web didáctica invitando a su utilización para la organización del estudio del tema. En el recurso web, el alumno puede encontrar una descripción detallada de los objetivos de cada tema y de sus elementos clave, orientaciones para su estudio, ejercicios de metacognición, cuestiones a resolver y diferentes documentos, cuidadosamente descritos, siempre con la intención de que el alumno pueda ir construyendo, de forma autónoma, sus propios materiales de estudio. La web didáctica incluye también descripción de actividades prácticas relacionadas con el temario de la asignatura, así como acceso a vídeos tutoriales, glosarios terminológicos o galerías de imágenes con las que completar su formación. Gracias a un cronograma detallado, el cual se va actualizando conforme va desarrollándose la asignatura, el estudiante puede ir avanzando en los contenidos que se presentarán de forma presencial, a la vez que le facilita la autogestión de su aprendizaje ya que en todo momento es consciente de la evolución de la asignatura y de los contenidos y las actividades que se van realizando. Por todo ello entendemos relevante que el estudiante acceda de forma regular a la web didáctica y 
actualice la información que de ella puede obtener, ya sea esta en forma de documentos de trabajo, actividades prácticas (presenciales o virtuales), etc.

Se han utilizado dos fuentes de datos para la realización de este estudio. El primero de ellos ha sido empleado para conocer el grado de implicación del estudiante con la web didáctica y el uso que hace de la misma. Para ello hemos usado los datos de registro estadístico ofrecidos desde la Plataforma Virtual de la Universidad de Murcia. Estos datos informan sobre los accesos que han realizado los estudiantes a los recursos web disponibles y empleados en su asignatura (número de visitas a la web de la asignatura, accesos a ficheros, tutorías, etc.). En nuestro estudio se contabilizaron el número total de accesos individuales de cada alumno a la página inicial de la web didáctica.

En segundo lugar, para poder valorar la relación entre la implicación del estudiante con el recurso web y sus resultados académicos, el profesorado que imparte las tres asignaturas participantes en este trabajo facilitó las puntuaciones finales obtenidas por los estudiantes en la primera convocatoria de la asignatura.

\subsection{Procedimiento.}

A fin de evitar posibles sesgos en la información aportada por los estudiantes originados por diferencias en el empleo y estructura de webs didácticas realizadas por distintos profesores, para este estudio se utilizaron tres asignaturas impartidas por el mismo docente cuyas webs didácticas mantenían un diseño común y eran empleadas como parte fundamental de una misma metodología presencial apoyada por las TIC, ya descrita en el apartado anterior.
En todos los casos, la información ha sido analizada respetando el anonimato de los participantes. Con este propósito se dispuso un código de identificación para cada uno de los estudiantes, común para los dos registros empleados, cuyos datos han sido cruzados en el estudio: número de accesos del alumno a la web didáctica de la asignatura y nota final del alumno en dicha asignatura en primera convocatoria.

La información referida al número de accesos que cada estudiante había realizado a la web didáctica se recopiló una vez finalizadas las asignaturas, asegurando de este modo que los estudiantes hubieran podido utilizar la web didáctica hasta el último momento.

\subsection{Diseño de la investigación y análisis de datos.}

El diseño de la investigación es cuantitativo no experimental, principalmente de corte descriptivo-correlacional (Hernández Pina \& Maquilón, 2010). Lo elegimos por considerarlo adecuado a los objetivos de la investigación. No obstante, no hemos renunciado a emplear técnicas de ANOVA para analizar algunos resultados concretos.

Dada la amplitud del rango en el número de accesos a la web didáctica y el elevado grado de dispersión de los mismos y con la finalidad de facilitar la comprensión de los resultados, hemos considerado adecuado aglutinar estos valores en intervalos. Siguiendo el procedimiento estadístico de cálculo de los mismos para la realización de histogramas de frecuencia, se han distribuido los valores de número de accesos a la web didáctica en torno a 14 intervalos, creando una nueva variable a la cual denominaremos Intervalo de accesos, con un total de 14 accesos en 


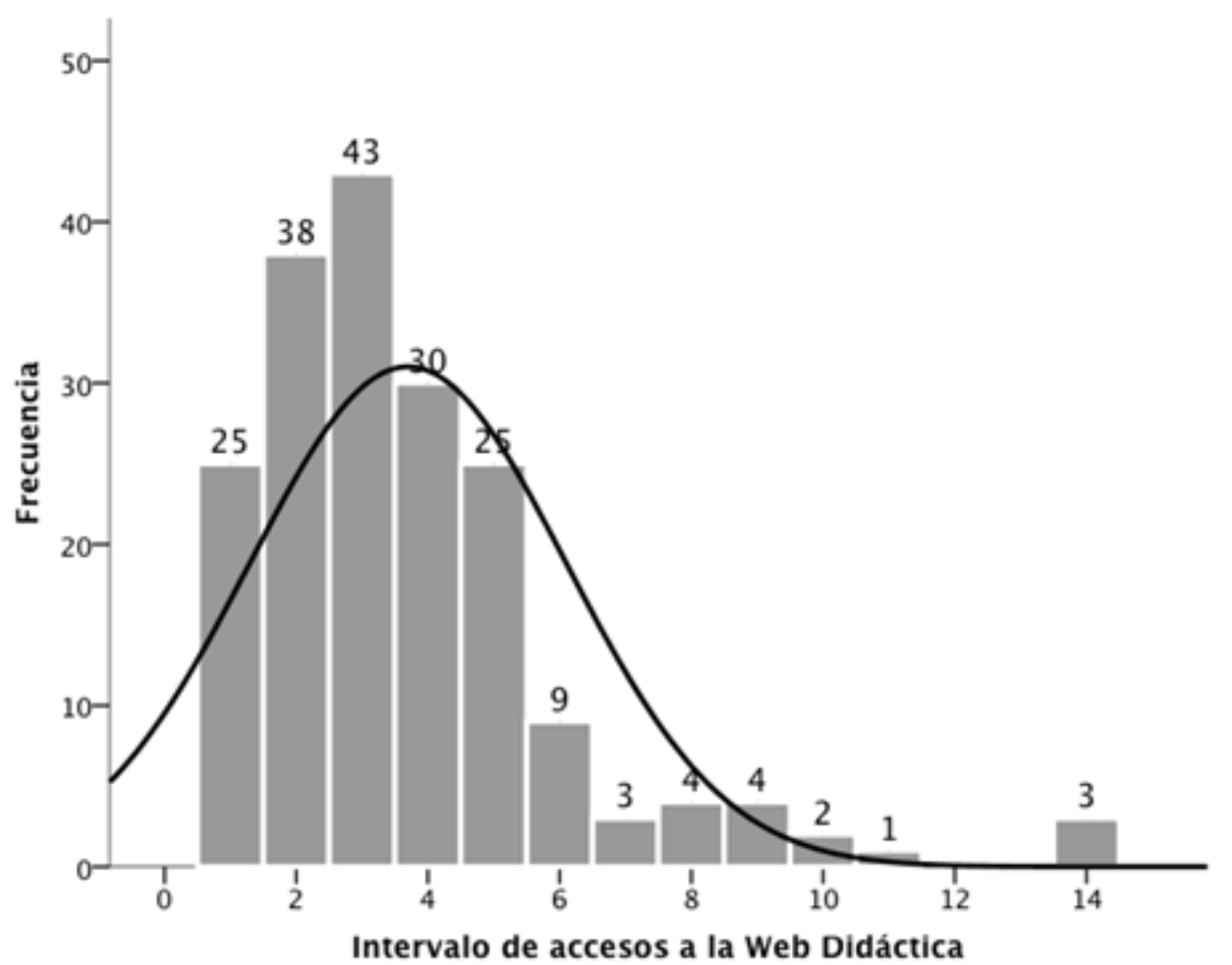

Figura 1. Frecuencias para los intervalos de accesos a la web didáctica.

cada intervalo. De tal forma que el intervalo 1 incluye los alumnos que han realizado entre 1 y 14 accesos; el intervalo 2 incluye aquellos alumnos que han accedido entre 14 y 28 veces, y así sucesivamente hasta el intervalo 14 que incluye a los alumnos que han realizado entre 182 y 198 accesos a la web didáctica. Los valores de accesos (296 y 470 accesos) correspondientes a dos alumnos fueron eliminados de los análisis por considerarse valores extremos y fuera del rango.

En el caso de las notas finales obtenidas por los alumnos, hemos establecido los cuatro intervalos de distribución clásica (suspenso de 0 a 4.9; aprobado de 5 a 6.9; notable de 7 a 8.9; y sobresaliente de 9 a 10) creando la variable que hemos denominado Rendimiento académico.

Para analizar el número de accesos a la web didáctica (puntuaciones directas y agrupadas en intervalos), a nivel global y en función de la variable rendimiento académico, hemos realizado un análisis descriptivo en el que calculamos los siguientes estadísticos: media, desviación típica, frecuencia y porcentaje.

Para comprobar la relación existente entre el número de accesos y la nota final, ambos agrupados en intervalos, hemos realizado el 
estadístico correlación de Spearman para variables ordinales.

Para identificar la existencia de diferencias significativas entre las medias hemos calculado ANOVA de un factor y sus correspondientes pruebas pos hoc.

Los datos recogidos fueron analizados a través del paquete estadístico SPSS (versión 17).

\section{Resultados.}

El objeto de este estudio es analizar la relación existente entre la implicación de los estudiantes con la web didáctica y su rendimiento académico. Por ello, en primer lugar realizamos una descripción a nivel global de los datos obtenidos, para pasar posteriormente a analizar los resultados para cada uno de los grupos de alumnos formados en función de las notas finales obtenidas en la asignatura correspondiente.

En la Figura 1 se presenta una distribución de las frecuencias de acceso de los estudiantes a las webs didácticas agrupadas en los 14 intervalos de accesos, permitiendo distinguir el número de estudiantes que ha coincidido en cada intervalo.

Como puede observarse, el $86.1 \%$ de los alumnos se sitúan dentro de los cinco primeros intervalos, los cuales incluyen a los estudiantes que han accedido a la web didáctica entre una y setenta veces, quedando el 14\% distribuido entre los intervalos restantes. Dado que los accesos a la web didáctica se han registrado a lo largo de 14 semanas lectivas, la media de accesos de los alumnos que se distribuyen entre los cinco intervalos de mayor frecuencia es de 3.2 visitas semanales por alumno a la web didáctica. No existen alumnos que hayan accedido a la web didáctica en los intervalos
12 y 13, que cubren la franja que oscila de 154 a 182 accesos a dichas webs, mientras que sólo el $1.6 \%$ de los alumnos se sitúan en el último intervalo (de 182 a 196 accesos). El mayor número de alumnos (43 estudiantes) lo encontramos en el tercer intervalo (de 28 a 42 accesos), lo que supone un $23 \%$ del total de participantes seguido del $20.3 \%$ de alumnos situados en el segundo intervalo que oscila entre 14 y 28 accesos. La media global de accesos a la web didáctica se encuentra en 44.4 accesos $(\mathrm{Sd} .=33.9)$, quedando situada entre el intervalo tres y cuatro (lo que supone una media $=3.7$ accesos semanales a la web didáctica; $\mathrm{Sd}=2.4$ ).

A continuación presentamos de forma gráfica los intervalos de accesos donde se sitúan los alumnos en función de los cuatro niveles de la variable rendimiento académico. Téngase en cuenta que, debido a que se trata de grupos heterogéneos en número de integrantes, en los resultados que presentamos a continuación estamos utilizando escalas diferentes para el eje de ordenadas (variable frecuencia de alumnos) en cada una de las gráficas que se incluyen en la Figura 2.

En el primer nivel de rendimiento académico encontramos un total de 24 estudiantes con una calificación inferior a 5 (suspenso), lo que supone el $12.8 \%$ de los participantes. La media de accesos totales a la web didáctica ofrecida por este grupo es de 36.3 accesos $(\mathrm{Sd} .=32.8)$ a lo largo del cuatrimestre (lo que supondría un promedio de 2.6 accesos por semana). Tal y como se aprecia en la Figura 2, el 75\% de los estudiantes de este nivel de rendimiento académico se agrupa en los tres primeros intervalos que oscilan desde uno hasta 42 accesos, quedando el $25 \%$ restante repartido entre distintos intervalos. Destacamos que no existen estudiantes que hayan accedido 

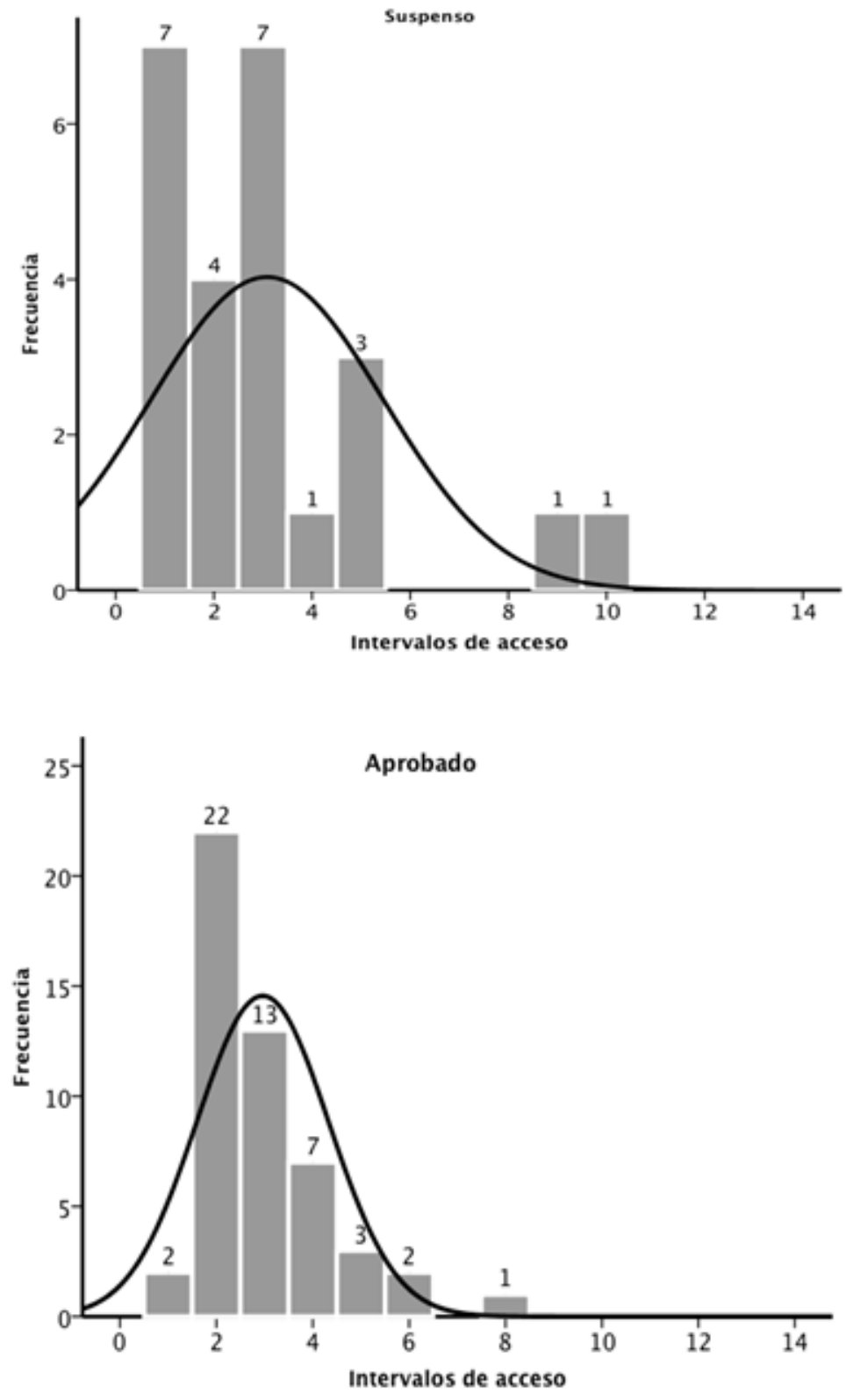

Figura 2. Frecuencia de intervalos de acceso a la web didáctica por niveles de la variable rendimiento académico.

\section{- 176 -}

Píxel-Bit. Revista de Medios y Educación. No 44. Enero 2014. ISSN: 1133-8482.

E-ISSN: 2171-7966. doi: http://dx.doi.org/10.12795/pixelbit.2014.i44.12 

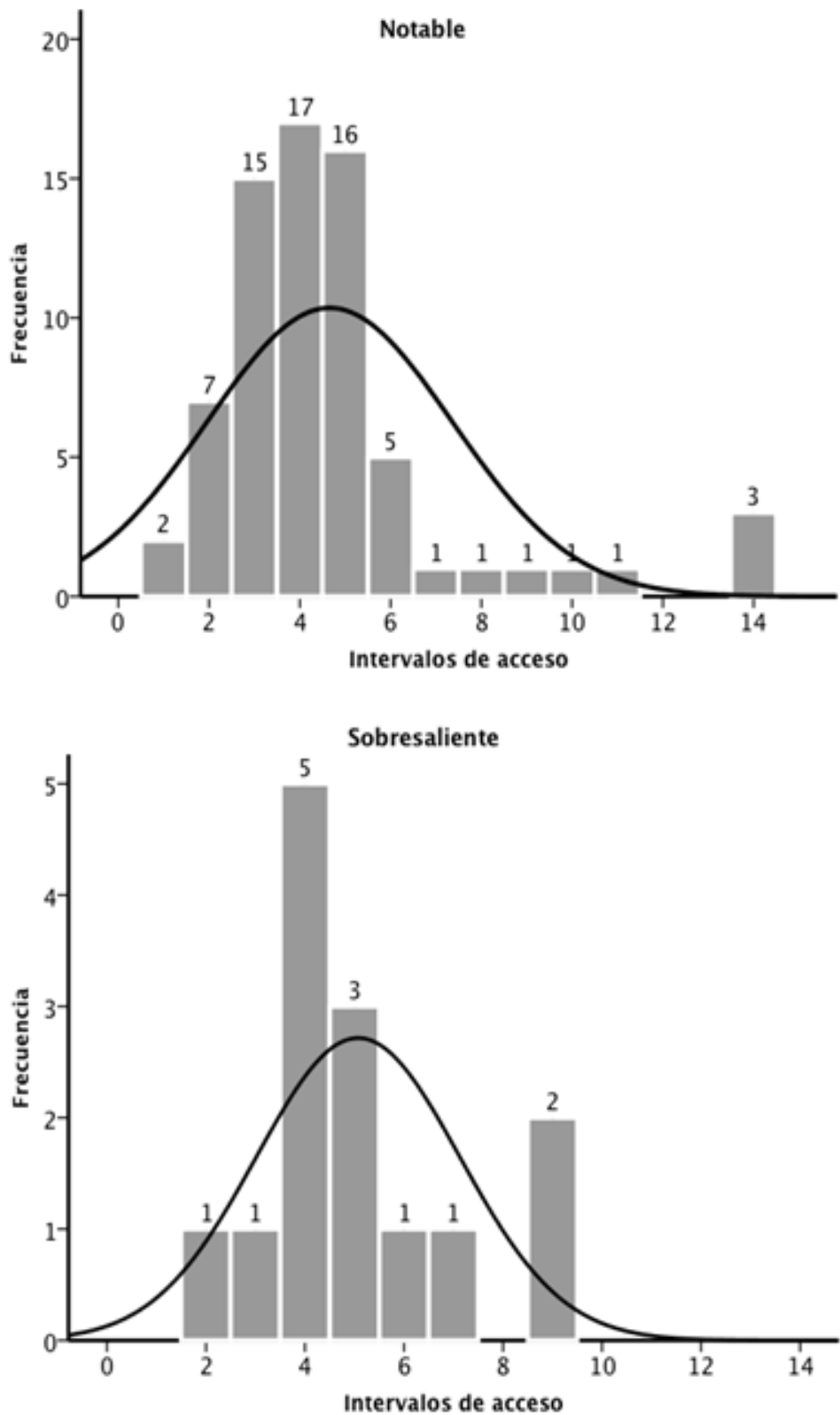

Figura 2. Frecuencia de intervalos de acceso a la web didáctica por niveles de la variable rendimiento académico.

$-177-$

Píxel-Bit. Revista de Medios y Educación. $N^{o}$ 44. Enero 2014. ISSN: 1133-8482.

E-ISSN: 2171-7966. doi: http://dx.doi.org/10.12795/pixelbit.2014.i44.12 
entre 70 y 112 veces, correspondiente a los intervalos seis, siete y ocho, encontrando, de forma aislada, en los intervalos nueve y diez (de 112 a 140 accesos) un $8.3 \%$ de los estudiantes ( $\mathrm{f}=2$ ), lo cual condiciona el valor medio de acceso a la web didáctica en este nivel de rendimiento académico y explica el elevado valor alcanzado por su desviación típica.

El segundo nivel de rendimiento académico es el compuesto por los estudiantes que obtuvieron notas entre 5 y 6.9 (aprobado). Está compuesto por 50 alumnos (el $26.7 \%$ de la muestra), con una media de accesos totales a la web didáctica de $34.6(\mathrm{Sd}=18.1)$. Esta media de accesos supondría un promedio de 2.5 visitas semanales. En la segunda gráfica de la Figura 2 como el primer intervalo de accesos cuenta con un número menor de estudiantes que en el caso de los estudiantes con calificación de suspenso, aglutinándose el $84 \%$ de los alumnos entre el segundo y el cuarto intervalo de accesos a la web didáctica (de 14 a 56 accesos) y encontrando menor dispersión de los alumnos en los diferentes intervalos de accesos.

El tercer nivel de rendimiento académico está compuesto por los alumnos cuya nota final fue de notable, es decir, que obtuvieron una calificación académica que oscilaba entre 7 y 8.9 puntos. Incluye a 70 alumnos, lo que supone el $37.4 \%$ de los participantes en el estudio. La media de accesos totales realizados por los alumnos que componen este grupo fue de 58.3 accesos $(\mathrm{Sd} .=38.0)$, mayor que la media ofrecida por los dos grupos de alumnos con rendimiento académico inferior. Esta media de accesos supone en el cuatrimestre un promedio de 4.2 visitas semanales a la web didáctica. Como se puede observar en la tercera gráfica de la Figura 2, más del $68 \%$ de estudiantes de calificación notable se sitúa entre los intervalos tres, cuatro y cinco (de 28 a 70 accesos), ampliándose este porcentaje al $85.7 \%$ si tomamos el intervalo dos (de 14 a 28 accesos) y el seis (de 70 a 84 accesos). De este grupo cabe destacar que es la agrupación de estudiantes que tiene mayor distribución en cuanto a los distintos intervalos, contando con al menos un estudiante en 12 de los 14 intervalos de accesos considerados, siendo también el único que tiene estudiantes (4.3\%) que han accedido a la web didáctica en un total de más de 182 ocasiones, lo que supone una media de 13 accesos semanales a lo largo del cuatrimestre.

El grupo de estudiantes que obtuvieron la calificación de sobresaliente (calificación académica final superior a 9) está formado por un total de 14 alumnos, equivalente al $7.5 \%$ del total de participantes. La media de accesos totales a la web didáctica alcanzada por estos estudiantes es de 64.1 accesos $(\mathrm{Sd} .=28.2)$, cercana a la encontrada en los alumnos con calificación de notable, y por encima de los alumnos con calificaciones finales inferiores a 7. Dicha media supone un promedio de 4.6 accesos a la semana. Podemos observar en la cuarta gráfica de la Figura 2 cómo el $57 \%$ de los estudiantes de este grupo se sitúan en los intervalos cuatro y cinco (de 42 a 56 accesos), repartiéndose otro $28.4 \%$ de forma homogénea en los intervalos dos, tres, seis y siete $(7.1 \%$ de participantes para en uno de ellos), situándose el $14.3 \%$ restante en el intervalo nueve que recoge aquellos alumnos que accedieron entre 112 y 126 veces a la web didáctica.

En Figura 3 se puede observar en el grupo de estudiantes que han superado la asignatura el incremento progresivo de la media de accesos totales en función de la 


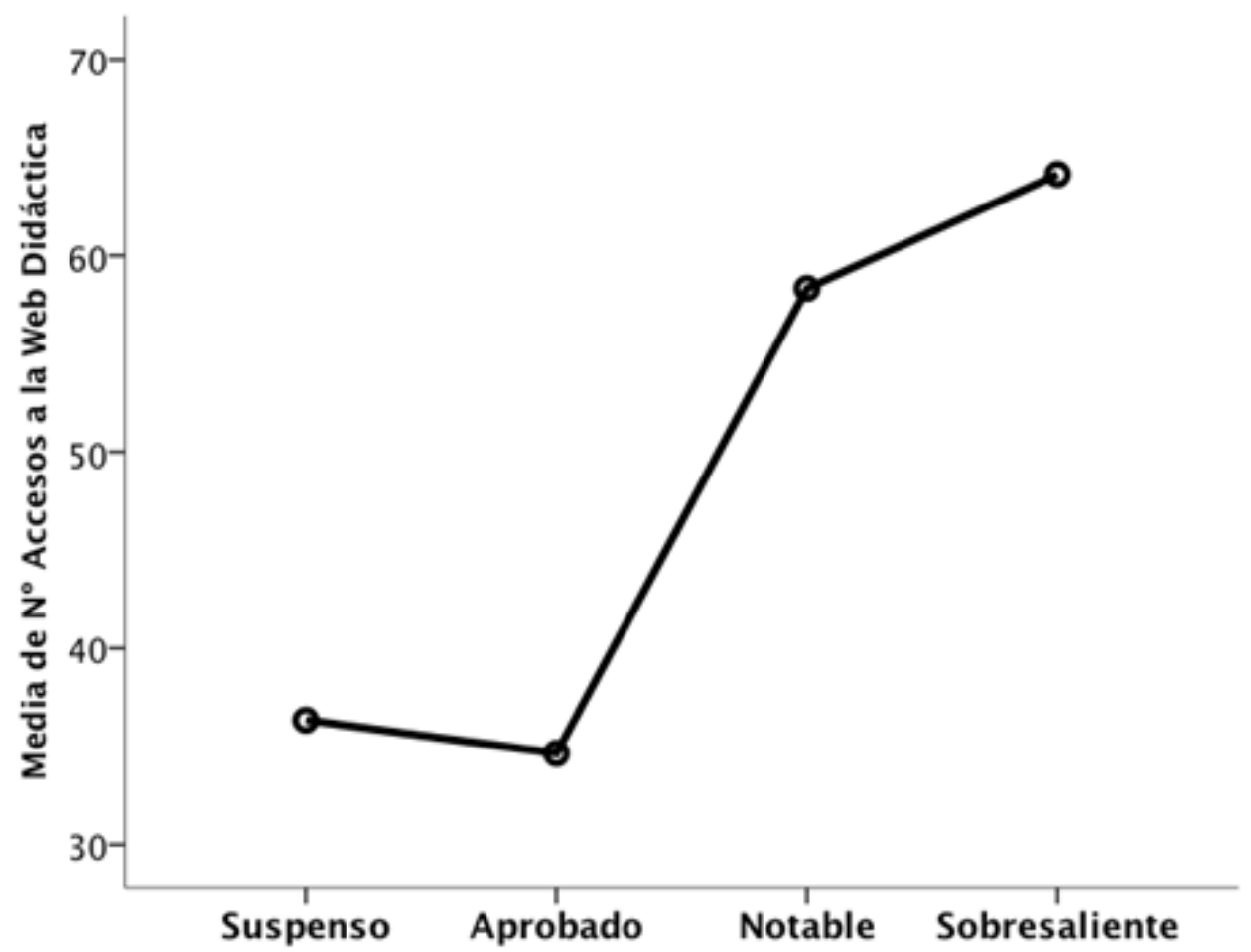

Figura 3. Valores medios de accesos totales a la web didáctica por calificación final.

calificación final obtenida. Se hace notar que, de acuerdo a la media de accesos de cada uno de los grupos de rendimiento académico, la media de los alumnos suspensos y aprobados se sitúa en el tercer intervalo de accesos de la Figura 1, mientras que la media de accesos de los alumnos con notable y sobresaliente los sitúa en el quinto intervalo

Se calculó el coeficiente de correlación de Spearman para variables ordinales entre los cuatro niveles de la variable rendimiento académico y los 14 niveles de la variable intervalo de accesos, encontrándose una correlación positiva $(r=.415$, Sig < .001) entre la calificación académica final obtenida por los estudiantes y su implicación en el acceso a la web didáctica.

Igualmente, un ANOVA de un factor realizado para la variable número de accesos totales a la web, utilizando como variable de agrupamiento la variable rendimiento académico, resultó significativo (F (3, 154) $=7.92$; Sig. <.001). Las pruebas pos hoc realizadas nos indican que la significación se debe a que los alumnos que obtuvieron la calificación de suspenso ( $\bar{x}=36.33$ accesos; $\mathrm{Sd}=32.80)$ presentaron significativamente menos accesos a la web que aquellos que aprobaron con notable $(\bar{x}=58.33$ accesos; 
$\mathrm{Sd}=38.04)(\mathrm{Sig}=.018) \mathrm{y} / \mathrm{o}$ con sobresaliente $(\bar{x}=64.14$ accesos; $\mathrm{Sd}=28.18)(\mathrm{Sig}=.045)$; a la vez, aquellos alumnos que aprobaron $(\bar{x}=34.64$ accesos; $\mathrm{Sd}=18.12)$ presentaron significativamente menos accesos que los que obtuvieron la calificación de notable $(\mathrm{Sig}<.000)$ o sobresaliente $(\mathrm{Sig}=.012)$.

\section{Discusión.}

Las TIC han cambiado la forma que tenemos de trabajar, de relacionarnos, y cómo no, también la manera que tenemos de acercarnos a la información, a la construcción del conocimiento y a la forma de transmitirlo. Aunque las TIC ya son una realidad en las aulas universitarias, las potencialidades, ventajas e implicaciones que pueden ofrecer a los procesos educativos son un tema pendiente de investigar en mayor profundidad.

Existe una amplia literatura que señala la valoración positiva que el alumnado hace de distintos recursos tecnológicos puestos al servicio del proceso de enseñanzaaprendizaje (Cabero et al., 2010; Chandra \& Ficher, 2009; Shahzad \& Khan, 2010; Tesone \& Ricci, 2008). También las webs didácticas han sido favorablemente valoradas, tanto por alumnos como por profesorado. Precisamente los alumnos, tras utilizar estas webs didácticas en sus asignaturas, han señalado que les han resultado útiles para su aprendizaje en la asignatura universitaria cursada, no solo para tener la documentación necesaria para cursar la asignatura, sino también para orientarse sobre cómo utilizarla y para realizar un aprendizaje autónomo. Los resultados de las experiencias de innovación con TIC llevadas a cabo por nuestro grupo de investigación en los últimos años nos han permitido considerar la web didáctica como un valioso recurso didáctico en los procesos de enseñanza (García Sánchez et al., 2010; García Sánchez \& Martínez Segura, 2009; Mirete, García Sánchez \& Sánchez López, 2011; Sánchez López et al., 2012). Pero, sobre todo, nos han llevado a estudiarlas como verdaderas herramientas cognitivas a disposición del aprendizaje. Hemos podido comprobar que con ella podemos generar procesos educativos de calidad, cuando son empleadas dentro de metodologías enfocadas a la construcción y autogestión del aprendizaje por parte del estudiante.

Los datos del presente estudio señalan, una vez más, que la web didáctica es una herramienta frecuentemente usada por los alumnos de la asignatura, con un promedio de algo más de tres visitas semanales por alumno, pudiendo interpretar que el estudiante se implica y hace uso del recurso didáctico en línea puesto a su disposición como apoyo a la docencia presencial. Pero además, en este estudio hemos podido comprobar empíricamente cómo la implicación que tienen los estudiantes con la web didáctica de una asignatura, medida por su volumen de accesos, se relaciona positivamente con sus resultados académicos. Así, el grado de implicación de los estudiantes con la web didáctica aumenta conforme va incrementándose la nota final obtenida por los mismos. Constatamos la existencia de una relación positiva entre mejor rendimiento académico y mayor implicación con la web didáctica. La única salvedad la encontramos en los alumnos que suspendieron la asignatura. Este grupo llegó a realizar un número medio de accesos a la web didáctica ligeramente mayor que el de alumnos que obtuvieron la calificación de aprobado. Pero esta leve diferencia pudo 
deberse claramente a la existencia de dos estudiantes suspensos que, alejándose de la tendencia del resto de alumnos con esa calificación, realizaron un número de accesos mucho más elevado que ellos, incrementando el valor de la media, pero elevando también de forma muy notoria la dispersión del grupo. Obviamente, la mera contabilización de los accesos no nos permite asegurar el uso adecuado del recurso y, en este caso, es posible interpretar que ese elevado número de accesos presentado por estos dos alumnos concretos, pudo estar respondiendo a un uso errático que no fue aprovechado para un adecuado aprendizaje. Pensamos, a modo incluso de hipótesis de trabajo para futuras investigaciones, que aquellos alumnos que han incluido las webs didácticas en sus procesos educativos, desarrollando estrategias de aprendizaje donde tienen cabida estas herramientas como un recurso más en el que apoyarse, posiblemente fueron los que obtuvieron mejores resultados académicos.

Lógicamente, no podemos afirmar que el mero hecho de acceder a la web didáctica sea la causa de la mejora en la nota final obtenida en la asignatura. Pero sí nos hace pensar que, quizás, los enfoques de aprendizaje de los estudiantes, las expectativas y la motivación que consigue el recurso o el ejercicio de metacognición que favorece, van a estar condicionando el uso que hacen de las webs didácticas y el resultado académico en la asignatura. Desde esta perspectiva, creemos posible considerar, nuevamente como hipótesis para futuros trabajos de investigación, la web didáctica como un recurso útil y válido para la educación superior, que podría integrarse dentro de las variables de presagio descritas por Biggs (1993) en su Modelo General de
Aprendizaje (Modelo 3P) sobre el que surge el constructo de los enfoques de aprendizaje.

Hemos constatado que los alumnos que han superado la asignatura con una nota de aprobado, junto con los suspensos, son los que menos han empleado las webs didácticas. Dadas las características de las webs didácticas empleadas, el requerimiento de uso que de ellas se hace en las asignaturas donde se emplean y los resultados obtenidos en este estudio, llegamos a la conclusión de que los alumnos cuyo objetivo fue cumplir con las exigencias de la asignatura pero empleando para ello un mínimo de esfuerzo, son aquellos que muestran una menor implicación con la web didáctica y, por tanto, un menor volumen de accesos a la herramienta. Esta implicación ha ido aumentando en proporción a la nota final obtenida por los estudiantes. Así, los que obtuvieron la calificación de notable o sobresaliente presentan un mayor número de accesos a la web que los aprobados o los suspensos, lo que interpretamos también como una mayor implicación con el uso de este recurso docente. Al mismo tiempo, los grupos que obtienen una mayor nota presentan una menor dispersión en sus accesos a la herramienta online, es decir, que parecen hacer un uso más consciente del recurso web empleándolo para obtener mejores resultados académicos.

Futuros estudios podrán retomar la teoría de los enfoques de aprendizaje (Biggs, 1987, 1993) para analizar si la mayor o menor implicación del alumno con la web didáctica está relacionado con enfoques de aprendizaje profundos o superficiales y con el desarrollo de estrategias para el estudio de la asignatura más adecuadas que les llevan a aprovechar mejor estos recursos puestos a su disposición. 
A modo de conclusión podemos señalar que, en nuestra opinión, las TIC en general y las webs didácticas en particular, pueden ser un factor determinante en la mejora de los resultados académicos de los estudiantes. La web didáctica permite ofrecer al alumno diversidad de recursos, adecuadamente orientados, con los cuales éste puede desarrollar un aprendizaje autónomo, así como estrategias de aprendizaje basadas en sus intereses académicos. Y esto nos parece especialmente interesante cuando nuestros estudiantes pertenecen a una era digital y para ellos conviene que diseñemos nuevos procesos educativos y herramientas de enseñanza más acordes con sus necesidades, intereses y formas de aprender.

\section{Fuentes de financiación.}

El Proyecto de Innovación de título Diseño de Webs-Home para asignaturas del Departamento de Métodos de Investigación y Diagnóstico en Educación, fue llevado a cabo durante el curso 2009/2010 y estuvo financiado por el Vicerrectorado de Relaciones de Relaciones Internacionales e Innovación de la Universidad de Murcia.

\section{Referencias bibliográficas.}

Area, M. (2003). Guía Didáctica. Internet en la docencia universitaria. webs docentes y aulas virtuales. Recuperado de http:// cedus.cl/files/guiadidacticawebs.pdf (2007). Algunos principios para el desarrollo de buenas prácticas pedagógicas con las TIC en el aula. Comunicación y pedagogía: Nuevas tecnologías y recursos didácticos, 222, 4247.
Biggs, J.B. (1987). Study Process Questionnaire (SPQ) Manual. Melburne: Australian Council for Educational Research. (1993). What do inventories of students' learning processes really measure? A theoretical review and clarification. British Journal of Educational Psychology, 63(1), 3-19. doi: 10.1111/j.2044-8279.1993.tb01038.x

Cabero, J. \& López, E. (2009). Evaluación de materiales multimedia en red en el Espacio Europeo de Educación Superior (EEES). Barcelona: Davinci.

Llorente, C. \& Puentes, A. (2010). La satisfacción de los estudiantes en red en la formación semipresencial. Comunicar, 35, 149-157. doi: 10.3916/C352010-03-08

Chandra, V. \& Fisher, D.L. (2009). Students' perceptions of a blended web-based learning environment. Learning Environ Res, 12, 3144. doi: 10.1007/s10984-008-9051-6

Domingo, M. \& Fuentes, M. (2010). Innovación educativa: experimentar con las TIC y reflexionar sobre su uso. Píxel-Bit. Revista de Medios y Educación, 36, 171-180. Recuperado de http://www.sav.us.es/pixelbit/ pixelbit/articulos/n36/13.pdf

García Sánchez, F.A. \& Martínez Segura, M.J. (2009). Web-docente y aprendizaje: una experiencia en el contexto de la convergencia al EEES. En R. Roig Vila (Dir.). Investigar desde un contexto educativo innovador. (pp. 201217). Alcoy: Marfil.

Mirete, A.B. \& Martínez-Juárez, M. (2010). Diseño de Web-s-Home para asignaturas del Departamento de Métodos de Investigación y Diagnóstico en Educación. En P. Arnaiz, L. Hernández \& M.P. García (Coords.). Experiencias de innovación educativa en la Universidad de Murcia. (pp. 115-141). Murcia: EDITUM. 
Garrison, D.R. \& Vaughan, N.D. (2008). Blended learning in higher education. Framework, principles and guidelines. San Francisco: Jossey-Bass.

Hernández Pina, F. \& Maquilón, J.J. (2010). Introducción a los diseños de investigación educativa. En S. Nieto (Ed.). Principios, métodos y técnicas esenciales para la investigación educativa. (pp. 109-126). Madrid: Dykinson.

Marín, V. \& Reche, E. (2011). La alfabetización digital del alumnado que accede a la Universidad de Córdoba. Edutec. Revista Electrónica de Tecnología Educativa, 35. Recuperado de http:// edutec.rediris.es/relevec2/relevec35

Marqués, P. (2005). Las webs docentes. Recuperado de http://dewey.uab.es/ pmarques/webdocente.htm

Mirete, A.B., García Sánchez, F.A. \& Sánchez López, M.C. (2011). Implicación del alumnado en la valoración de su satisfacción con las Webs Didácticas. Edutec. Revista Electrónica de Tecnología Educativa, 37, 1-13. Recuperado de http://edutec.rediris.es/ $\mathrm{R}$ e v e 1 e c $2 / \mathrm{R}$ e v e 1 e c $37 /$ implicacion_alumnado_valoracion _satisfacion_webs_didacticas.html.

Shahzad, A.H. \& Khan, A. (2010). Virtual learning and students perception-a research study. Procedia Social and Behavioral Sciences, 2, 5463-5467. http://dx.doi.org/ 10.1016/j.sbspro.2010.03.891

Snart, J.A. (2010). HybridLearning. Theperils and promise of blending online and face-to-face instruction in higher education. California: ABC CLIO.

Sánchez López, C., García Sánchez, F.A., Martínez Segura, M.J. \& Mirete, A.B. (2012). Aproximación a la valoración que el alumnado hace de recursos online utilizados para la docencia universitaria. Píxel-Bit. Revista de
Medios y Educación, 40(1), 35-45. Recuperado de http://acdc.sav.us.es/pixelbit/ images/stories/p40/03.pdf

Tejedor, F.J., García-Valcárcel, A. \& Parada, S. (2009). Medida de actitudes del profesorado universitario hacia la integración de las TIC. Comunicar, 33, 115-124. doi: 10.3916/c332009-03-002

Tesone, D.V. \& Ricci, P. (2008). Student perceptions of Web-based instruction: a comparative análisis. MERLOT Journal of Online Learning and Teaching, 4(3), 317-324.

Fecha de recepción: 24-07-2012

Fecha de evaluación: 07-10-2012

Fecha de aceptación: 27-12-2012 